Bull. Austral. Math. Soc.

53 в 25,53 в 20

VOL. 66 (2002) [465-475]

\title{
FROM SURFACES IN THE 5-SPHERE TO 3-MANIFOLDS IN COMPLEX PROJECTIVE 3-SPACE
}

\author{
J. Bolton, C. Scharlach and L. VRancken
}

In a previous paper it was shown how to associate with a Lagrangian submanifold satisfying Chen's equality in 3-dimensional complex projective space, a minimal surface in the 5-sphere with ellipse of curvature a circle. In this paper we focus on the reverse construction.

\section{INTRODUCTION}

It was proved in [7] that at each point $p$ of a totally real submanifold $M^{n}$ of a holomorphic space form $\widetilde{M}(4 c)$ of constant holomorphic sectional curvature $4 c$ we have

$$
\delta_{M}(p) \leqslant \frac{n^{2}(n-2)}{2(n-1)} H^{2}(p)+\frac{1}{2}(n+1)(n-2) c,
$$

where $H$ denotes the length of the mean curvature vector and $\delta_{M}$ is the Riemannian invariant introduced by Chen in [6], defined by

$$
\delta_{M}(p)=\tau(p)-(\inf K)(p) .
$$

Here

$$
(\inf K)(p)=\inf \left\{K(\pi) \mid \pi \text { is a } 2 \text {-dimensional subspace of } T_{p} M\right\}
$$

where $K(\pi)$ is the sectional curvature of $\pi$, and $\tau(p)=\sum_{i<j} K\left(e_{i} \wedge e_{j}\right)$ denotes the scalar curvature defined in terms of an orthonormal basis $\left\{e_{1}, \ldots, e_{n}\right\}$ of the tangent space $T_{p} M$.

Then $M^{n}$ is said to satisfy Chen's equality if equality is attained in (1) for each $p \in M$. In the case where $n=3$ and the surrounding space is $\mathbb{C}^{3}$ this corresponds to one of the classes of Lagrangian submanifolds studied by Bryant in [5].

In a previous paper [2] we gave a local construction which associated to a Lagrangian submanifold satisfing Chen's equality but having no totally geodesic points in complex

\footnotetext{
Received 29th April, 2002

We are grateful to the London Mathematical Society and the University of Utrecht for financial support during the preparation of this paper.

Much of the early work in connection with this paper was carried out in collaboration with our late, and very much missed, colleague Dr L.M. Woodward of the University of Durham. We are pleased to acknowledge his contribution.
}

Copyright Clearance Centre, Inc. Serial-fee code: 0004-9727/02 \$A2.00+0.00. 
projective space $\mathbb{C} P^{3}(4)$, a minimal surface in $S^{5}(1)$ with ellipse of curvature a circle. In this paper, we focus on the reverse construction.

In Section 2 we consider the case in which a minimal surface with ellipse of curvature a circle is contained in a totally geodesic $S^{4}(1)$ of $S^{5}(1)$. The immersion is then superminimal [4], and our construction in this case is based on the well known correspondence [4] between superminimal surfaces in $S^{4}(1)$ and horizontal holomorphic curves in $\mathbb{C} P^{3}(4)$.

In Section 3, which is the main part of the paper, we consider the case of a linearly full minimal surface in $S^{5}(1)$ whose ellipse of curvature is a circle. Here we use the theory of harmonic sequences to show how to construct locally a submanifold $M^{3}$ of $S O(6)$ whose Maurer-Cartan equations coincide with equations (9) to (14) of Section 4 of [2]. Then, since $S U(4)$ is a double cover of $S O(6)$, we obtain a local lift into $S U(4)$ for which projection onto the first column defines a Lagrangian immersion of $M^{3}$ into $\mathbb{C} P^{3}(4)$ satisfying Chen's equality. It will be apparent that the constructions described in this paper provide a local inverse of the construction described in [2].

\section{SUPERMinimal SURfaces IN $S^{4}(1)$}

In this section we assume that $N^{2}$ is an oriented surface superminimally immersed in $S^{4}(1)$. The orientation, together with the metric induced on $N^{2}$, enables us to give $N^{2}$ the structure of a Riemann surface in such a way that the immersion is conformal.

We first recall the following result of Bryant [4] relating superminimal immersions of $N^{2}$ into $S^{4}(1)$ to holomorphic horizontal immersions of $N^{2}$ into $\mathbb{C} P^{3}(4)$.

Theorem 1. (Bryant) Let $T: \mathbb{C} P^{3}(4) \rightarrow S^{4}(1)$ be the twistor fibration and let $\phi: N^{2} \rightarrow S^{4}(1)$ be a superminimal immersion of a simply connected Riemann surface. Then there exists a unique horizontal holomorphic immersion $\widetilde{\phi}: N^{2} \rightarrow \mathbb{C} P^{3}(4)$ such that $T \circ \widetilde{\phi}= \pm \phi$.

Conversely if $\tilde{\phi}: N^{2} \rightarrow \mathbb{C} P^{3}(4)$ is a horizontal holomorphic curve, then $T \circ \tilde{\phi}: N^{2}$ $\rightarrow S^{4}(1)$ is a (possibly branched) superminimal immersion.

Now, let $\tilde{\phi}: N^{2} \rightarrow \mathbb{C} P^{3}(4)$ be a horizontal holomorphic curve defined on a simply connected Riemann surface $N^{2}$ and let $p_{i}: S^{7}(1) \rightarrow \mathbb{C} P^{3}(4)$ denote the Hopf fibration determined by the complex structure on $\mathbb{R}^{8}=\mathbb{C}^{4}$ given by multiplication by $i$. It is clear that the natural immersion $\psi$ of the pullback bundle $M^{3}=\tilde{\phi}^{*}\left(S^{7}(1)\right)$, defined so that the following diagram commutes, is invariant (and hence minimal) in the Sasakian space form $\left(S^{7}(1), I,\langle.,\rangle.\right)$. Here, $I$ is the Sasakian structure determined on $S^{7}(1)$ by multiplication by $i$ on $\mathbb{R}^{8}=\mathbb{C}^{4}$.

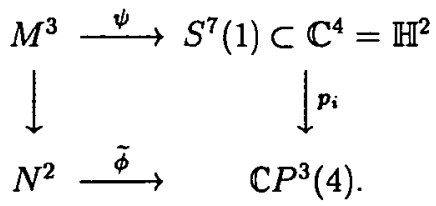


In fact, we may use multiplication by $i, j, k$ on $\mathbb{R}^{8}=\mathbb{H}^{2}$ to define corresponding Hopf fibrations of $S^{7}(1)$ over $\mathbb{C} P^{3}(4)$, and we let $p_{j}: S^{7}(1) \rightarrow \mathbb{C} P^{3}$ be the one determined by multiplication by $j$. Since $\tilde{\phi}$ is horizontal and holomorphic, the immersion $\psi$ is horizontal with respect to $p_{j}[\mathbf{1}]$ and so we may apply the following special case of a theorem of Reckziegel [9].

THEOREM 2. (Reckziegel) Let $\psi: M^{3} \rightarrow S^{7}(1) \subset \mathbb{C}^{4}$ be an immersion which is horizontal with respect to the Hopf fibration $p_{j}: S^{7}(1) \rightarrow \mathbb{C} P^{3}$. Then $p_{j} \psi: M^{3}$ $\rightarrow \mathbb{C} P^{3}(4)$ is a Lagrangian immersion which is minimal if and only if $\psi$ is minimal.

Conversely, let $\widetilde{\psi}: M^{3} \rightarrow \mathbb{C} P^{3}(4)$ be a Lagrangian immersion of a connected, simply connected manifold $M^{3}$. Then there exists a map $\psi: M^{3} \rightarrow S^{7}(1)$, which is horizontal with respect to $p_{j}$, such that $p_{j} \psi=\tilde{\psi}$. Moreover, any two such lifts $\psi_{1}$ and $\psi_{2}$ are related by $\psi_{2}=e^{i \theta} \psi_{1}$ where $\theta$ is a constant.

Hence, combining the above two theorems, we see that starting from a superminimal immersion $\phi: N^{2} \rightarrow S^{4}(1)$, we obtain a minimal Lagrangian immersion $p_{j} \psi: M^{3}$ $\rightarrow \mathbb{C} P^{3}(4)$. Note that $i \psi$ is tangential to the immersion $\psi$ of $M^{3}$ into $S^{7}(1)$, and if $D$ denotes the standard flat connection on $\mathbb{R}^{8}$ then for $X$ tangential to $M$,

$$
D_{X}(i \psi)=i D_{X} \psi=i X
$$

Hence if $h$ denotes the second fundamental form of $\psi$ in $S^{7}(1)$, we see that $h(., i \psi)=0$. It then follows from [7] and [8] that $p_{j} \psi: M^{3} \rightarrow \mathbb{C} P^{3}(4)$ satisfies Chen's equality. Moreover, it is clear that if we apply the construction of $[2]$ to $p_{j} \psi$ we recover the immersion $\phi$.

\section{LiNEARLy fUll MiNimal SURFACES IN $S^{5}(1)$}

Let $f: N^{2} \rightarrow S^{5}(1)$ be a minimal immersion of an oriented surface. As in Section 2, we use the orientation and induced metric to give $N^{2}$ the structure of a Riemann surface in such a way that $f$ is a conformal immersion. If $I I$ denotes the second fundamental form of $f$ in $S^{5}$ we recall that the image under $I I$ of the unit circle in a tangent space of $N^{2}$ is a (possibly degenerate) ellipse called the ellipse of curvature.

From now on, we assume that $f: N^{2} \rightarrow S^{5}(1)$ is a linearly full minimal immersion of an oriented surface with ellipse of curvature a non-degenerate circle at each point. We now show how to locally associate to such an immersion a unitary moving frame. The approach we follow here is based on the theory of harmonic sequences, which we describe briefly below for the special case of minimal surfaces in $S^{5}(1)$ with ellipse of curvature a circle. The reader is referred to [3] for more details in the general situation of minimal surfaces in $S^{m}(1)$ or $\mathbb{C} P^{m}(4)$.

Let $z=x+i y$ be a local complex coordinate on $N^{2}$, and denote $\frac{\partial}{\partial z}$ by $\partial$ and $\frac{\partial}{\partial \bar{z}}$ 
by $\bar{\partial}$. We introduce $\mathbb{C}^{6}$-valued functions $f_{0}, f_{1}, f_{2}$ by

$$
\begin{aligned}
& f_{0}=f \\
& f_{1}=\partial f \\
& f_{2}=I I(\partial, \partial),
\end{aligned}
$$

where $I I$ now denotes the complex bilinear extension of the second fundamental form of $f$ in $S^{5}(1)$. If $(, \quad)$ is the complex bilinear extension of the standard inner product on $\mathbb{R}^{6}$, it follows that $\left(f_{0}, f_{1}\right)=0$ while conformality of $f$ is equivalent to

$$
\left(f_{1}, f_{1}\right)=0 \text {. }
$$

Thus $f_{0}, f_{1}, \bar{f}_{1}$ are mutually unitarily orthogonal and $f_{2}$ is the component of $\partial f_{1}$ unitarily orthogonal to $f_{0}, f_{1}, \bar{f}_{1}$.

If $f_{2}=a-i b$ where $a, b$ are $\mathbb{R}^{7}$ valued functions then, using minimality of $f$,

$$
I I\left(\cos \phi \frac{\partial}{\partial x}+\sin \phi \frac{\partial}{\partial y}, \cos \phi \frac{\partial}{\partial x}+\sin \phi \frac{\partial}{\partial y}\right)=2(a \cos 2 \phi+b \sin 2 \phi),
$$

so that the ellipse of curvature is a circle if and only if

$$
f_{2} \neq 0 \text { and }\left(f_{2}, f_{2}\right)=0 \text {, }
$$

so that in this case $f_{2}$ and $\bar{f}_{2}$ are unitarily orthogonal. Hence, $f_{0}, f_{1}, \bar{f}_{1}, f_{2}, \bar{f}_{2}$ are mutually unitarily orthogonal non-zero vectors.

Finally, we define $f_{3}$ to be the component of $\partial f_{2}$ which is unitarily orthogonal to $\left\{f_{0}, f_{1}, \bar{f}_{1}, f_{2}, \bar{f}_{2}\right\}$. As the immersion is contained in $S^{5}(1)$, we deduce that $f_{3}$ and $\bar{f}_{3}$ are linearly dependent.

By Takahashi's Lemma, the minimality condition for $f$ may be written as $\partial \bar{\partial} f_{0}$ $=\lambda f_{0}$ for some $\lambda \in \mathbb{R}$, and an inductive argument readily shows that if we put $w_{p}$ $=\log \left|f_{p}\right|, \quad p=1,2,3$, then

$$
\begin{aligned}
& \partial f_{0}=f_{1}, \\
& \partial f_{1}=f_{2}+2 \partial w_{1} f_{1}, \\
& \partial f_{2}=f_{3}+2 \partial w_{2} f_{2},
\end{aligned}
$$

while

$$
\begin{aligned}
& \bar{\partial} f_{1}=-e^{2 w_{1}} f_{0}, \\
& \bar{\partial} f_{2}=-e^{2\left(w_{2}-w_{1}\right)} f_{1} \\
& \bar{\partial} f_{3}=-e^{2\left(w_{3}-w_{2}\right)} f_{2}
\end{aligned}
$$

So far, everything is valid for an arbitrary choice of local complex coordinate but we now pick a special coordinate to facilitate calculations. It follows from (12) that 
$\left(\bar{\partial} f_{3}, f_{3}\right)=0$, so that $\left(f_{3}, f_{3}\right) d z^{6}$ is a holomorphic differential on $N^{2}$. Hence, away from the isolated points at which $f_{3}=0$, we can choose a local complex coordinate $z$ for which

$$
\left(f_{3}, f_{3}\right)=1
$$

so that

$$
f_{3} \text { is real and } w_{3}=0 .
$$

We now introduce a unitary moving frame $\left\{F_{0}, \ldots, F_{5}\right\}$ by setting $F_{0}=f_{0}, F_{1}$ $=e^{-w_{1}} f_{1}, F_{2}=e^{-w_{2}} f_{2}, F_{3}=f_{3}, F_{-1}=-\bar{F}_{1}$ and $F_{-2}=\bar{F}_{2}$ (the minus sign in the definition of $F_{-1}$ is there for reasons connected with the theory of harmonic sequences, and makes no essential difference in the present paper). A straightforward computation shows that

$$
\begin{aligned}
& d F_{0}=e^{w_{1}} d z F_{1}-e^{w_{1}} d \bar{z} F_{-1} \\
& d F_{1}=-e^{w_{1}} d \bar{z} F_{0}+\left(\partial w_{1} d z-\bar{\partial} w_{1} d \bar{z}\right) F_{1}+e^{w_{2}-w_{1}} d z F_{2}, \\
& d F_{-1}=e^{w_{1}} d z F_{0}+\left(-\partial w_{1} d z+\bar{\partial} w_{1} d \bar{z}\right) F_{-1}-e^{w_{2}-w_{1}} d \bar{z} F_{-2}, \\
& d F_{2}=-e^{w_{2}-w_{1}} d \bar{z} F_{1}+\left(\partial w_{2} d z-\bar{\partial} w_{2} d \bar{z}\right) F_{2}+e^{-w_{2}} d z F_{3} \\
& d F_{-2}=e^{w_{2}-w_{1}} d z F_{-1}+\left(-\partial w_{2} d z+\bar{\partial} w_{2} d \bar{z}\right) F_{-2}+e^{-w_{2}} d \bar{z} F_{3}, \\
& d F_{3}=-e^{-w_{2}} d \bar{z} F_{2}-e^{-w_{2}} d z F_{-2} .
\end{aligned}
$$

We now consider the manifold $W$ of unitary frames $\left\{V_{0}, V_{1}, V_{-1}, V_{2}, V_{-2}, V_{3}\right\}$ of the form

$$
\left\{V_{0}, V_{1}, V_{-1}, V_{2}, V_{-2}, V_{3}\right\}=\left\{F_{0}, e^{i \alpha} F_{1}, e^{-i \alpha} F_{-1}, e^{i \beta} F_{2}, e^{-i \beta} F_{-2}, F_{3}\right\}, \quad \alpha, \beta \in \mathbb{R} .
$$

Thus, we may regard $W$ as the bundle of strongly adapted unitary frames over $N^{2}$, in that $V_{1}$ (respectively $V_{2}$ ) spans the $(1,0)$ component of the complexified tangent space (respectively first normal space) of $N^{2}$. If we use $z=x+i y, \alpha$ and $\beta$ as local coordinates on $W$, it follows easily from (15)-(20) that

$$
\begin{aligned}
& d V_{0}=e^{w_{1}-i \alpha} d z V_{1}-e^{w_{1}+i \alpha} d \bar{z} V_{-1} \\
& d V_{1}=-e^{w_{1}+i \alpha} d \bar{z} V_{0}+\left(\partial w_{1} d z-\bar{\partial} w_{1} d \bar{z}+i d \alpha\right) V_{1}+e^{w_{2}-w_{1}-i(\beta-\alpha)} d z V_{2}, \\
& d V_{-1}=e^{w_{1}-i \alpha} d z V_{0}+\left(-\partial w_{1} d z+\bar{\partial} w_{1} d \bar{z}-i d \alpha\right) V_{-1}-e^{w_{2}-w_{1}+i(\beta-\alpha)} d \bar{z} V_{-2}, \\
& d V_{2}=-e^{w_{2}-w_{1}-i(\alpha-\beta)} d \bar{z} V_{1}+\left(\partial w_{2} d z-\bar{\partial} w_{2} d \bar{z}+i d \beta\right) V_{2}+e^{-w_{2}+i \beta} d z V_{3} \\
& d V_{-2}=e^{w_{2}-w_{1}+i(\alpha-\beta)} d z V_{-1}+\left(-\partial w_{2} d z+\bar{\partial} w_{2} d \bar{z}-i d \beta\right) V_{-2}+e^{-w_{2}-i \beta} d \bar{z} V_{3} \\
& d V_{3}=-e^{-w_{2}-i \beta} d \bar{z} V_{2}-e^{-w_{2}+i \beta} d z V_{-2} .
\end{aligned}
$$

We now wish to compare the above formulae to those obtained in Section 4 of [2]. We recall that there, with a Lagrangian submanifold $M^{3}$ of $\mathbb{C} P^{3}$ satisfying Chen's equality but having no totally geodesic points, we locally associated a smooth map $\left\{U_{0}, \ldots, U_{5}\right\}$ : $M^{3} \rightarrow S O(6)$ such that 
(i) the image of $U_{0}$ is a minimal surface in $S^{5}(1)$,

(ii) $U_{1}$ and $U_{2}$ span the tangent space to this surface,

(iii) $U_{3}$ and $U_{4}$ span the first normal space to this surface,

(iv) $U_{5}$ is the remaining orthogonal vector such that $\operatorname{det}\left(U_{0}, \ldots, U_{5}\right)=1$.

We now write

$$
\begin{aligned}
& \tilde{U}_{0}=U_{0} \\
& \tilde{U}_{1}=\frac{1}{\sqrt{2}}\left(U_{1}-i \varepsilon_{1} U_{2}\right), \\
& \tilde{U}_{-1}=-\frac{1}{\sqrt{2}}\left(U_{1}+i \varepsilon_{1} U_{2}\right), \\
& \widetilde{U}_{2}=\frac{1}{\sqrt{2}}\left(U_{3}-i \varepsilon_{2} U_{4}\right), \\
& \tilde{U}_{-2}=\frac{1}{\sqrt{2}}\left(U_{3}+i \varepsilon_{2} U_{4}\right), \\
& \widetilde{U}_{3}=U_{5}
\end{aligned}
$$

where $\varepsilon_{1}, \varepsilon_{2}= \pm 1$ will be chosen later. If we now rewrite equations (9)-(14) of Section 4 of [2] with respect to this frame, we find that for suitably chosen functions $a, b, c, d$ and orthonormal basis $\left\{\theta_{1}, \theta_{2}, \theta_{3}\right\}$ of local 1 -forms on $M$ we have

$$
\begin{aligned}
& d \widetilde{U}_{0}=b_{10} \widetilde{U}_{1}+b_{-10} \widetilde{U}_{-1} \text {, } \\
& d \tilde{U}_{1}=-\bar{b}_{10} \widetilde{U}_{0}+i \varepsilon_{1}\left(c \theta_{1}+d \theta_{2}+\left(1-\frac{1}{3} b\right) \theta_{3}\right) \tilde{U}_{1}+b_{21} \widetilde{U}_{2}+b_{-21} \widetilde{U}_{-2}, \\
& d \widetilde{U}_{-1}=-\bar{b}_{-10} \widetilde{U}_{0}-i \varepsilon_{1}\left(c \theta_{1}+d \theta_{2}-\left(1-\frac{1}{3} b\right) \theta_{3}\right) \tilde{U}_{-1}+b_{2-1} \widetilde{U}_{2}+b_{-2-1} \widetilde{U}_{-2}, \\
& d \tilde{U}_{2}=-\bar{b}_{21} \widetilde{U}_{1}-\bar{b}_{2-1} \widetilde{U}_{-1}+i \varepsilon_{2}\left(c \theta_{1}+d \theta_{2}-\left(1+\frac{1}{3} b\right) \theta_{3}\right) \widetilde{U}_{2}+b_{32} \tilde{U}_{3}, \\
& d \tilde{U}_{-2}=-\bar{b}_{-21} \tilde{U}_{1}-\bar{b}_{-2-1} \tilde{U}_{-1}-i \varepsilon_{2}\left(c \theta_{1}+d \theta_{2}-\left(1+\frac{1}{3} b\right) \theta_{3}\right) \tilde{U}_{-2}+b_{3-2} \tilde{U}_{3}, \\
& d \widetilde{U}_{3}=-\bar{b}_{32} \widetilde{U}_{2}-\bar{b}_{3-2} \widetilde{U}_{-2}
\end{aligned}
$$

where there exists a positive function $\lambda$ such that

$$
\begin{aligned}
& b_{10}=-\bar{b}_{-10}=\frac{1}{\sqrt{2}}\left(-a \theta_{1}+(1+b) \theta_{2}\right)-i \frac{\varepsilon_{1}}{\sqrt{2}}\left((1+b) \theta_{1}+a \theta_{2}\right), \\
& b_{21}=-\bar{b}_{-2-1}=\frac{1}{2} \lambda\left(\left(1-\varepsilon_{1} \varepsilon_{2}\right) \theta_{1}+i\left(\varepsilon_{1}-\varepsilon_{2}\right) \theta_{2}\right), \\
& b_{-21}=-\bar{b}_{2-1}=\frac{1}{2} \lambda\left(\left(1+\varepsilon_{1} \varepsilon_{2}\right) \theta_{1}+i\left(\varepsilon_{1}+\varepsilon_{2}\right) \theta_{2}\right), \\
& b_{32}=\bar{b}_{3-2}=\frac{1}{\sqrt{2}}\left(a \theta_{1}+(1-b) \theta_{2}\right)+i \frac{\varepsilon_{2}}{\sqrt{2}}\left((1-b) \theta_{1}-a \theta_{2}\right) .
\end{aligned}
$$


We now find a hypersurface $\widehat{M}^{3}$ of the manifold $W$ of strongly adapted unitary frames over $N^{2}$ described above, together with linearly independent local 1-forms $\theta_{1}, \theta_{2}$, $\theta_{3}$, and local functions $\lambda>0, a, b, c, d$ defined on $\widehat{M}^{3}$, such that the systems (21)-(26) and (27)-(32) of differential equations coincide.

So, assume that $a_{\ell k}$ (respectively $b_{\ell k}$ ) are the components of $d V_{k}$ (respectively $d \widetilde{U}_{k}$ ) in the direction of $V_{\ell}$ (respectively $\widetilde{U}_{\ell}$ ). As $a_{-21}=0$, it follows that we need $b_{-21}=0$ and thus

$$
\varepsilon_{1} \varepsilon_{2}=-1
$$

Next, we find that if we require that

$$
\begin{aligned}
b_{21} & =a_{21}, \\
b_{10}+b_{32} & =a_{10}+a_{32},
\end{aligned}
$$

then we need that

$$
\begin{aligned}
\lambda\left(\theta_{1}+i \varepsilon_{1} \theta_{2}\right) & =e^{w_{2}-w_{1}+i(\alpha-\beta)} d z \\
\sqrt{2}\left(\theta_{2}-i \varepsilon_{1} \theta_{1}\right) & =\left(e^{w_{1}-i \alpha}+e^{-w_{2}+i \beta}\right) d z
\end{aligned}
$$

Hence, we see that the positive function $\lambda$ must satisfy

$$
\lambda\left(e^{w_{1}-i \alpha}+e^{-w_{2}+i \beta}\right)+\sqrt{2} i \varepsilon_{1} e^{w_{2}-w_{1}+i(\alpha-\beta)}=0
$$

which, as $\lambda$ is real, implies that

$$
\lambda\left(e^{w_{1}+i \alpha}+e^{-w_{2}-i \beta}\right)-\sqrt{2} i \varepsilon_{1} e^{w_{2}-w_{1}-i(\alpha-\beta)}=0 .
$$

It follows from the two previous equations that the following conditions need to be satisfied:

$$
\begin{aligned}
& 0 \neq\left(e^{w_{1}-i \alpha}+e^{-w_{2}+i \beta}\right), \\
& \lambda=-\sqrt{2} i \varepsilon_{1} \frac{e^{w_{2}-w_{1}+i(\alpha-\beta)}}{\left(e^{w_{1}-i \alpha}+e^{-w_{2}+i \beta}\right)} \\
& e^{w_{2}} \cos (2 \alpha-\beta)+e^{-w_{1}} \cos (2 \beta-\alpha)=0,
\end{aligned}
$$

where $\varepsilon_{1}= \pm 1$ is determined by the requirement that $\lambda$ be positive.

LEMMA 1. The conditions (36) and (38) determine a hypersurface $\widehat{M}^{3}$ of $W$, which may be parametrized by $z$ and $t=\alpha+\beta$.

Proof: We first introduce new coordinates $s$ and $t$ on $W$ by

$$
\begin{aligned}
& s=\alpha-\beta \\
& t=\alpha+\beta
\end{aligned}
$$


Then (38) becomes

$$
e^{w_{2}} \cos \left(\frac{1}{2} t+\frac{3}{2} s\right)+e^{-w_{1}} \cos \left(\frac{1}{2} t-\frac{3}{2} s\right)=0
$$

which we can rewrite as

$$
\left(e^{w_{2}}+e^{-w_{1}}\right) \cos \left(\frac{1}{2} t\right) \cos \left(\frac{3}{2} s\right)=\left(e^{w_{2}}-e^{-w_{1}}\right) \sin \left(\frac{1}{2} t\right) \sin \left(\frac{3}{2} s\right) .
$$

It then follows that

$$
\cot \left(\frac{3}{2} s\right)=\frac{e^{w_{1}+w_{2}}-1}{e^{w_{1}+w_{2}}+1} \tan \frac{1}{2} t
$$

To determine $s$ explicitly in terms of $t$ (up to an initial condition), we differentiate (39) with respect to $t$ and find that

$$
s^{\prime}(t)=-\frac{1}{3} \frac{e^{2\left(w_{1}+w_{2}\right)}-1}{e^{2\left(w_{1}+w_{2}\right)}+1+2 e^{\left(w_{1}+w_{2}\right)} \cos t} .
$$

The denominator of the right hand side vanishes only if $w_{1}+w_{2}=0$ and $t=(2 k+1) \pi$, $k \in \mathbb{Z}$, which is excluded by (36). The function $s(t)$ is now determined (up to a addition of an integer multiple of $(2 \pi) / 3)$ by the condition that $\cos ((3 / 2) s)=0$ when $t$ is an integer multiple of $2 \pi$.

We now compute the 1 -forms $\theta_{1}, \theta_{2}, \theta_{3}$ and the function $\lambda$ on $\widehat{M}^{3}$. As $\lambda$ is real valued, we see using (37) that

$$
\begin{aligned}
\lambda^{2} & =\lambda \bar{\lambda} \\
& =\frac{2 e^{2\left(w_{2}-w_{1}\right)}}{\left(e^{w_{1}-i \alpha}+e^{-w_{2}+i \beta}\right)\left(e^{w_{1}+i \alpha}+e^{-w_{2}-i \beta}\right)} \\
& =\frac{2 e^{2\left(w_{2}-w_{1}\right)}}{e^{2 w_{1}}+e^{-2 w_{2}}+2 e^{w_{1}-w_{2}} \cos t} \\
& =\frac{e^{3\left(w_{2}-w_{1}\right)}}{\cosh \left(w_{1}+w_{2}\right)+\cos t} .
\end{aligned}
$$

Hence, as $\lambda$ is positive, it follows that

$$
\lambda=\frac{e^{3\left(w_{2}-w_{1}\right) / 2}}{\sqrt{\cosh \left(w_{1}+w_{2}\right)+\cos t}} .
$$

From (35), we obtain

$$
\sqrt{2}\left(\theta_{2}-i \varepsilon_{1} \theta_{1}\right)=\left(e^{w_{1}-(i(s+t)) / 2}+e^{-w_{2}+(i(t-s)) / 2}\right) d z
$$

which determines the 1 -forms $\theta_{1}$ and $\theta_{2}$. The 1 -form $\theta_{3}$ is determined by the condition that

$$
a_{11}+a_{22}=b_{11}+b_{22}
$$


Indeed, taking into (33) into account, it follows that

$$
\theta_{3}=-i \varepsilon_{1}\left(\partial\left(w_{1}+w_{2}\right) d z-\bar{\partial}\left(w_{1}+w_{2}\right) d \bar{z}\right)+\frac{1}{2} \varepsilon_{1} d t
$$

We may proceed in two different ways in order to obtain a Lagrangian immersion of $\widehat{M}^{3}$ into $\mathbb{C} P^{3}(4)$. The first possibility is to use the following existence and uniqueness result of $[8]$.

Theorem 3. Let $\left(M^{n},\langle.,\rangle.\right)$ be an $n$-dimensional simply connected Riemannian manifold. Let $\sigma$ be a symmetric bilinear vector-valued form on $M^{n}$ satisfying

(i) $\langle\sigma(X, Y), Z\rangle$ is totally symmetric,

(ii) $(\nabla \sigma)(X, Y, Z)=\nabla_{X} \sigma(Y, Z)-\sigma\left(\nabla_{X} Y, Z\right)-\sigma\left(Y, \nabla_{X} Z\right)$ is totally symmetric,

(iii) $R(X, Y) Z=\langle Y, Z\rangle X-\langle X, Z\rangle Y+\sigma(\sigma(Y, Z), X)-\sigma(\sigma(X, Z), Y)$.

Then there exists a Lagrangian isometric immersion $x:\left(M^{n},\langle.,\rangle.\right) \rightarrow \mathbb{C} P^{n}(4)$ such that the second fundamental form $h$ satisfies $h(X, Y)=J \sigma(X, Y)$. Moreover, $x$ is determined uniquely modulo holomorphic isometries of $\mathbb{C} P^{n}(4)$.

The above result may be applied in the following way. . We start with the minimal surface $N^{2}$ equipped with the special local complex coordinate $z$ chosen so that (13) holds. We consider the 3-dimensional manifold $\widehat{M}^{3}$ of $W$ constructed in Lemma 1 , but excluding those points where $w_{1}(z)+w_{2}(z)=0$ and $\cos t=-1$. We define 1 -forms $\theta_{1}$, $\theta_{2}$ and $\theta_{3}$ on $\widehat{M}^{3}$ using (42) and (43), where $\varepsilon_{1}$ is determined by the two equations (37) and (41) for $\lambda$ and the initial condition chosen for $s$. We denote the dual vector fields corresponding to these 1-forms by $E_{1}, E_{2}$ and $E_{3}$ and define a metric on $\widehat{M}^{3}$ by requiring that $E_{1}, E_{2}$ and $E_{3}$ form an orthonormal moving frame on $\widehat{M}^{3}$. We define a positive function $\lambda$ on $\widehat{M}^{3}$ by (41) and introduce a symmetric bilinear vector valued form $\sigma$ on $\widehat{M}^{3}$ by

$$
\begin{array}{ll}
\sigma\left(E_{1}, E_{1}\right)=\lambda E_{1}, & \sigma\left(E_{1}, E_{3}\right)=0 \\
\sigma\left(E_{1}, E_{2}\right)=-\lambda E_{2}, & \sigma\left(E_{2}, E_{3}\right)=0 \\
\sigma\left(E_{2}, E_{2}\right)=-\lambda E_{1}, & \sigma\left(E_{3}, E_{3}\right)=0 .
\end{array}
$$

It is then straighforward to compute that all the conditions of Theorem 3 are satisfied and hence there exists a Lagrangian immersion with the desired properties of $\widehat{M}^{3}$ into $\mathbb{C} P^{3}(4)$.

The second way to proceed is to continue with the comparison of the systems (21)(26) and (27)-(32) in order to determine the functions $a, b, c$ and $d$ explicitly. The requirement that

$$
a_{10}-a_{32}=b_{10}-b_{32}
$$


necessitates that

$$
\begin{aligned}
\left(e^{w_{1}-i \alpha}-e^{-w_{2}+i \beta}\right) d z & =-\sqrt{2}\left(a+i \varepsilon_{1} b\right)\left(\theta_{1}+i \varepsilon_{1} \theta_{2}\right) \\
& =-i \varepsilon_{1} \sqrt{2}\left(\theta_{2}-i \varepsilon_{1} \theta_{1}\right)\left(a+i \varepsilon_{1} b\right) \\
& =-i \varepsilon_{1}\left(a+i \varepsilon_{1} b\right)\left(e^{w_{1}-(i(s+t)) / 2}+e^{-w_{2}+\frac{1}{2} i(t-s)}\right) d z
\end{aligned}
$$

where we have used (42) for the final equality. Hence,

$$
\begin{aligned}
\left(a+i \varepsilon_{1} b\right) & =i \varepsilon_{1} \frac{\left(e^{w_{1}-(i(t+s)) / 2}-e^{-w_{2}+(i(t-s)) / 2}\right)}{\left(e^{w_{1}-(i(s+t)) / 2}+e^{-w_{2}+(i(t-s)) / 2}\right)} \\
& =i \varepsilon_{1} \frac{\left(e^{w_{1}+w_{2}}-e^{i t}\right)}{\left(e^{w_{1}+w_{2}}+e^{i t}\right)}
\end{aligned}
$$

which determines $a$ and $b$. Specifically, we have that

$$
\begin{aligned}
& a=2 \varepsilon_{1} \frac{e^{w_{1}+w_{2}} \sin t}{e^{2\left(w_{1}+w_{2}\right)}+1+2 e^{w_{1}+w_{2}} \cos t}, \\
& b=-\frac{1-e^{2\left(w_{1}+w_{2}\right)}}{e^{2\left(w_{1}+w_{2}\right)}+1+2 e^{w_{1}+w_{2}} \cos t} .
\end{aligned}
$$

Finally, in order to obtain $c$ and $d$, we consider the condition that

$$
a_{11}-a_{22}=b_{11}-b_{22} \text {. }
$$

This yields

$$
\partial\left(w_{1}-w_{2}\right) d z-\bar{\partial}\left(w_{1}-w_{2}\right) d \bar{z}+i d s=2 \varepsilon_{1}\left(c \theta_{1}+d \theta_{2}-\frac{1}{3} b \theta_{3}\right)
$$

or, equivalently,

$$
\begin{aligned}
\partial\left(w_{1}-w_{2}\right) d z & -\bar{\partial}\left(w_{1}-w_{2}\right) d \bar{z}-\frac{1}{3} i \frac{e^{2\left(w_{1}+w_{2}\right)}-1}{e^{2\left(w_{1}+w_{2}\right)}+1+2 e^{\left(w_{1}+w_{2}\right)} \cos t} d t \\
& =2 \varepsilon_{1}\left(c \theta_{1}+d \theta_{2}+\frac{1}{3} \frac{1-e^{2\left(w_{1}+w_{2}\right)}}{e^{2\left(w_{1}+w_{2}\right)}+1+2 e^{w_{1}+w_{2}} \cos t} \theta_{3}\right) .
\end{aligned}
$$

Using (43) the above equation gives

$$
\begin{aligned}
& \partial\left(w_{1}-w_{2}\right) d z-\bar{\partial}\left(w_{1}-w_{2}\right) d \bar{z} \\
& \quad=2 \varepsilon_{1}\left(c \theta_{1}+d \theta_{2}-\frac{1}{3} i \varepsilon_{1} \frac{1-e^{2\left(w_{1}+w_{2}\right)}}{e^{2\left(w_{1}+w_{2}\right)}+1+2 e^{w_{1}+w_{2}} \cos t}\left(\partial\left(w_{1}+w_{2}\right) d z-\bar{\partial}\left(w_{1}+w_{2}\right) d \bar{z}\right)\right) .
\end{aligned}
$$

However, it follows from (35) that $d z$ and $d \bar{z}$ may be expressed as linear combinations of $\theta_{1}$ and $\theta_{2}$, so that $c$ and $d$ are uniquely determined by the above equation. It is now straightforward to check that the systems (21)-(26) and (27)-(32) coincide. Therefore, using the double cover of $S O(6)$ by $S U(4)$ as described in Section 4 of [2], we obtain a Lagrangian immersion satisfying Chen's equality.

Again, it is clear that if we apply the construction of [2] to this Lagrangian immersion, we obtain the linearly full minimal immersion $f: N^{2} \rightarrow S^{5}(1)$ from which we started. 


\section{REFERENCES}

[1] D.E. Blair, F. Dillen, L. Verstraelen and L. Vrancken, 'Calabi curves as holomorphic Legendre curves and Chen's inequality', Kyungpook Math. J. 35 (1996), 407-416.

[2] J. Bolton, C. Scharlach, L. Vrancken and L.M. Woodward, 'From certain minimal Lagrangian submanifolds of the 3-dimensional complex projective space to minimal surfaces in the 5-sphere' (to appear), in Proceedings of the Fifth Pacific Rim Geometry Conference, Tohoku University to appear.

[3] J. Bolton and L.M. Woodward, 'Congruence theorems for harmonic maps from a Riemann surface into $\mathbb{C} P^{n}$ and $S^{n}$, J. London Math. Soc. (2) 45 (1992), 363-376.

[4] R.L. Bryant., 'Conformal and minimal immersions of compact surfaces into the 4-sphere', J. Differential Geom. 17 (1982), 455-473.

[5] R.L. Bryant., 'Second order families of special Lagrangian 3-folds', (preprint).

[6] B.-Y. Chen., 'Some pinching and classification theorems for minimal submanifolds', Arch. Math. 60 (1993), 568-578.

[7] B.-Y. Chen, F. Dillen, L. Verstraelen and L. Vrancken, 'An exotic totally real minimal immersion of $S^{3}$ in $\mathbb{C} P^{3}$ and its characterization', Proc. Royal Soc. Edinburgh 126 (1996), 153-165.

[8] B.-Y. Chen, F. Dillen, L. Verstraelen and L. Vrancken, 'Totally real submanifolds of $\mathbb{C} P^{n}$ satisfying a basic equality', Arch. Math. 63 (1994), 553-564.

[9] H. Reckziegel, 'Horizontal lifts of isometric immersions into the bundle space of a pseudo-Riemannian submersion', in Global Differential Geometry and Global Analysis (1984), Lecture Notes in Mathematics 1156 (Springer Verlag, Berlin, Heidelberg, New York, 1985), pp. 264-279.

Departmentof Mathematical Sciences

University of Durham

Durham DH1 3LE

United Kingdom

e-mail: john.bolton@dur.ac.uk
Fachbereich Mathematik, Sekr. MA 8-3

Technische Universität Berlin

Strasse des 17 Juni 136

10623 Berlin

Germany

e-mail: cs@sfb288.math.tu-berlin.de

Laboratoire de Mathématiques

LAMATH, ISTV2

Université de Valenciennes

59313 Valenciennes Cedex 9

France

e-mail: luc.vrancken@univ-valenciennes.fr 